\section{Advances in molecular profiling of malignant melanoma: ready for clinical practice?}

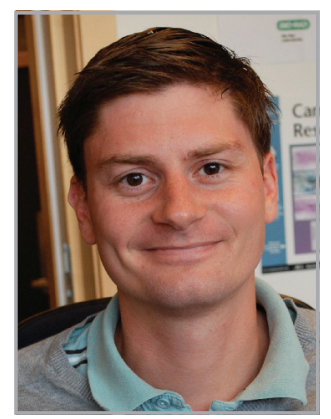

\author{
"A combination of mutation and gene expression \\ molecular profiling has great potential for future \\ individualized care of patients with melanoma."
}

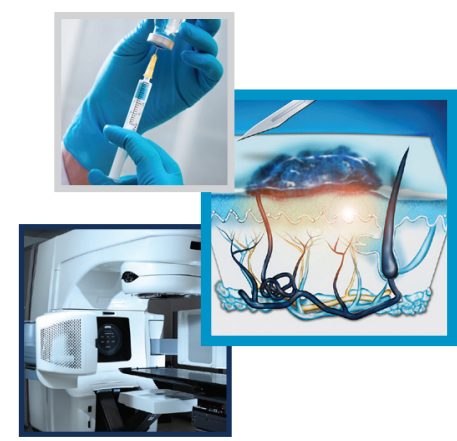

Göran Jönsson*

Cutaneous malignant melanoma (CMM) is one of the most common cancers in the western world, with a rapidly increasing incidence. Clinically, CMM is a highly heterogeneous disease that is currently divided according to the American Joint Committee on Cancer (AJCC) staging system [1]. Several tumor-intrinsic features such as Breslow thickness, ulceration and mitotic rate are incorporated in the AJCC staging system, representing well-established prognostic markers. CMM patients with T1 tumors $(<1 \mathrm{~mm}$ in Breslow thickness) have about $90 \%$ survival while those with T4 tumors (>4 $\mathrm{mm}$ in thickness) have a survival rate of approximately 40\% [1]. Despite the excellent prognosis within the T1 group, there have been efforts to further stratify these tumors using tumor infiltrating lymphocytes (TILs), mitogenicity and the AJCC variables [2]. Overall, this study shows that these features may add independent prognostic information in thin melanomas. However, a major challenge lies within stage II CMM, that is, lymph node negative patients with tumors having a Breslow thickness ranging from at least $1 \mathrm{~mm}$ to very large lesions $(>4 \mathrm{~mm})$, with or without ulceration. Survival for these patients varies considerably and refinement with additional prognostic biomarkers is needed.

Based on the discovery in 2002 of frequent BRAF mutations in CMM [3], and their virtually mutually exclusive occurrence with NRAS mutations, tumors are today molecularly divided by $B R A F$ and NRAS mutation status. Research in CMM was then focused on determining the therapeutic relevance of this oncogene. Previously, in 2010 the efficacy of selective BRAF kinase inhibitors was demonstrated and subsequently reinforced by Phase II studies [4-6]. In all, BRAF mutation (most commonly Val600Glu) is now established as a strong predictor for BRAF inhibition treatment. BRAF mutation status is also one of the cornerstones in the CMM classification proposed by the group of Boris Bastian in 2005 [7]. They describe that $B R A F$ mutation is frequently found in CMMs that are intermittently sun-induced (e.g., melanomas located on the trunk), while tumors located on non-sun-induced sites (acral and mucosal melanomas) usually are $B R A F$ wild-type. Furthermore, $B R A F$ mutation has been proposed as a marker of poor prognosis in the distant
KEYWORDS

- BRAF - gene expression

- melanoma • mutation 


\section{“The aim of personalized medicine is to provide the right therapy for the right patient."}

metastatic setting [8,9]. More recently, wholeexome sequencing of melanoma tumors has been used to identify novel oncogenic drivers, the most prominent candidate being $R A C 1$, where a hotspot codon mutation was found in two separate studies $[10,11]$. The successful development of BRAF-targeted therapies has undoubtedly helped to introduce molecular classification in the clinical setting. Studies investigating the clinical relevance of novel gene mutations are awaited.

The scarcity, in comparison to other malignancies such a breast cancer, of studies investigating the correlation between gene expression (GEX)-based molecular signatures and clinical outcome in primary CMM cohorts is mainly due to the lack of frozen tumor tissue from patients with sufficient clinical follow-up. An early study of 58 primary CMM identified a 254-GEX signature associated with 4-year distant metastasisfree survival [12]. The signature was significantly associated with Breslow thickness, AJCC stage, type of melanoma, ulceration and mitotic rate, all of which are strong established prognostic features in melanoma. Furthermore, the authors were able to correctly classify 11 out of 17 CMM with respect to their known clinical outcome. Subsequently, the authors tested 23 proteins from the 254-gene classifier in an independent set of 176 primary CMM using immunohistochemistry. Two proteins (MCM4 and MCM6) were found to have a significant impact on prognosis even after adjusting for Breslow thickness, ulceration, age and sex. However, even though this study initiated a GEX-based classification of melanoma, subsequent validation studies are still lacking.

Technical development has allowed researchers to determine GEX in formalin-fixed, paraffin-embedded tissue. In melanoma, the group of Julia Newton-Bishop spearheaded this field with the analysis of RNA levels in 502 cancer genes $[13,14]$. Specifically, the group analyzed 254 primary CMM specimens and found that expression of DNA repair genes (RAD52 and TOP2A) were significant predictors of relapse-free survival in multivariable models. The involvement of DNA repair genes and prognosis in CMM is further supported by the Winnenpenenckx et al. study [12].

In contrast, several groups have analyzed GEX profiles from metastatic CMM [15-18]. A distinct common denominator in these studies is the finding that elevated expression of immune response-related genes is associated with improved outcome. Bogunovic et al. defined a GEX signature in stage III CMM consisting mainly of immune response genes and tested its predictive capacity against the AJCC staging system, TILs, CD3 positivity and mitotic index. Although mitotic index was the most significant predictor, the GEX signature did indeed harbor independent predictive capacity in a multivariable analysis [15]. Further support for the importance of immune response GEX signatures in metastatic CMM came from a study by Mann et al. where a GEX signature together with $B R A F$ and $N R A S$ mutations predicted poor outcome in macroscopic stage III melanomas [18].

In an attempt to define molecular subtypes analogous to the more established breast cancer GEX subtypes used in clinical practice [19], we used unsupervised analysis of tumors from 57 metastatic CMM [17]. Importantly, this cohort consisted of stage IV patients enrolled in a clinical trial investigating the effect of dacarbizine (DTIC). The GEX patterns identified by the unsupervised analysis highlighted DNA repair, cell cycle, pigmentation and immune response as the dominant features of the profiles. Four GEX subtypes were identified and consequently named high immune response, proliferative, pigmentation and normal-like. Importantly, a significant correlation to outcome was seen, with patients of the proliferative subtype having the worst survival. Although $B R A F$ and NRAS mutations were found in all subtypes, there was a tendency of more mutations in the proliferative subtype. Homozygous deletions of the CDKN2A locus were also preferentially found in the proliferative subtype, whereas brisk infiltration of CD3-positive cells was frequently found in highimmune response metastatic CMM, which is associated with better survival. Consequently, this suggests that the GEX subtypes have distinct intrinsic molecular properties. The finding that immune response GEX signatures correlate with improved patient outcome is captivating in the light of the new era of immunotherapies such as anti-CTLA4 and anti-PD1 antibodies in melanoma treatment. Such a GEX signature may be correlated with benefit from immunotherapy and, thus, of direct clinical impact. Indeed, a recent study of patient undergoing MAGE-A3 immunotherapy demonstrated a benefit in cases with increased expression of, for example, T-cellassociated genes [20]. Moreover, similar observations were done in ipilimumab-treated patients 
[21] suggesting that the composition of the tumor microenvironment play an important role in the antitumor activity of immunotherapy.

The prognostic impact of the molecular subtypes, established in metastatic CMM, was reinforced in a follow up study of 223 primary CMM. Again, high-immune response classified melanomas displayed an improved relapse-free survival while pigmentation and proliferative classified primary CMMs had a poor outcome. Based on the molecular subtypes, a prognostic classifier was designed that divided primary melanomas into two groups (low and high grade). This prognostic classifier showed independent prognostic capacity in a multivariable model and was further validated in the Winnenpeninckx et al. dataset [22]. Finally, the signature showed prognostic power in the more clinically challenging primary CMM (Breslow thickness $>2 \mathrm{~mm}$ ). Additional testing of the signature and subsequent clinical implementation would aid in refinement of, in particular, stage II melanoma.

Notably, based on the results from these two studies $[17,22]$ only $7 \%$ of primary melanomas were classified as proliferative, while in the stage IV melanomas 21\% were proliferative, suggesting that the distribution of subtypes is dependent on disease stage. More recently, we have also observed that multiple metastases from the same patient can be of different molecular subtype [23]. This emphasizes that molecular events in the metastatic process is complex, which also may explain the intrinsic ability of melanomas to develop resistance to therapies. This complexity is further supported by the finding of acquired genetic alterations such as NRAS, MEK2 and PTEN mutations in BRAF inhibitor-resistant tumors [24].

\section{References}

1 Balch CM, Gershenwald JE, Soong SJ et al. Final version of 2009 AJCC melanoma staging and classification. J. Clin. Oncol. 27, 6199-6206 (2009)

2 Gimotty PA, Elder DE, Fraker DL et al. Identification of high-risk patients among those diagnosed with thin cutaneous melanomas. J. Clin. Oncol. 25, 1129-1134 (2007).

3 Davies H, Bignell GR, Cox C et al. Mutations of the BRAF gene in human cancer. Nature 417, 949-954 (2002).

4 Flaherty KT, Puzanov I, Kim KB et al. Inhibition of mutated, activated $B R A F$ in

An obvious limitation of hitherto performed GEX profiling studies in metastatic melanoma is that they are derived from patients that did not receive state-of-the art treatment, therapies that would have markedly changed their outcome. Therefore, we do not know whether the results of these GEX signatures are applicable in patients treated with BRAF inhibition, anti-CTLA4 or anti-PD1 even though an immune response signature may suggest benefit of immune therapy. The aim of personalized medicine is to provide the right therapy for the right patient. Much progress has been made in BRAF-mutated melanoma during the past few years with the introduction of several highly efficient and selective kinase inhibitors. However, it is time to consider the next step, that is, an improved understanding of each individual melanoma tumor. Somatic mutation analysis of $B R A F$ has become routine clinical practice in melanoma and screening for other gene mutations will follow. Now, additional validation and testing of GEX-based tumor classification in the clinical setting is required. A combination of mutation and GEX molecular profiling has great potential for future individualized care of patients with melanoma.

\section{Financial \& competing interests disclosure}

The author has no relevant affiliations or financial involvement with any organization or entity with a financial interest in or financial conflict with the subject matter or materials discussed in the manuscript. This includes employment, consultancies, honoraria, stock ownership or options, expert testimony, grants or patents received or pending, or royalties.

No writing assistance was utilized in the production of this manuscript.

metastatic melanoma. N. Engl. J. Med. 363, 809-819 (2010).

5 Chapman PB, Hauschild A, Robert C et al. Improved survival with vemurafenib in melanoma with $B R A F \mathrm{~V} 600 \mathrm{E}$ mutation. N. Engl. J. Med. 364, 2507-2516 (2011).

6 Sosman JA, Kim KB, Schuchter L et al. Survival in BRAF V600-mutant advanced melanoma treated with vemurafenib. $N$. Engl. J. Med. 366, 707-714 (2012).

7 Curtin JA, Fridlyand J, Kageshita T et al. Distinct sets of genetic alterations in melanoma. N. Engl. J. Med. 353, 2135-2147 (2005).

8 Ekedahl H, Cirenajwis H, Harbst K et al. The clinical significance of $B R A F$ and $N R A S$
"... it is time to consider the next step, that is, an improved understanding of each individual melanoma tumor.” mutations in a clinic-based metastatic melanoma cohort. Br. J. Dermatol. 169, 1049-1055 (2013).

9 Long GV, Menzies AM, Nagrial AM et al. Prognostic and clinicopathologic associations of oncogenic $B R A F$ in metastatic melanoma. J. Clin. Oncol. 29, 1239-1246 (2011).

10 Hodis E, Watson IR, Kryukov GV et al. A landscape of driver mutations in melanoma. Cell 150, 251-263 (2012).

11 Krauthammer M, Kong Y, Ha BH et al. Exome sequencing identifies recurrent somatic RAC1 mutations in melanoma. Nat. Genet. 44, 1006-1014 (2012).

12 Winnepenninckx V, Lazar V, Michiels S et al. Gene expression profiling of primary 
cutaneous melanoma and clinical outcome. J. Natl Cancer Inst. 98, 472-482 (2006).

13 Conway C, Mitra A, Jewell R et al. Gene expression profiling of paraffin-embedded primary melanoma using the DASL assay identifies increased osteopontin expression as predictive of reduced relapse-free survival. Clin. Cancer Res. 15, 6939-6946 (2009).

14 Jewell R, Conway C, Mitra A et al. Patterns of expression of DNA repair genes and relapse from melanoma. Clin. Cancer Res. 16, 5211-5221 (2010).

15 Bogunovic D, O’Neill DW, Belitskaya-Levy I et al. Immune profile and mitotic index of metastatic melanoma lesions enhance clinical staging in predicting patient survival. Proc. Natl Acad. Sci. USA 106, 20429-20434 (2009).

16 John T, Black MA, Toro TT et al. Predicting clinical outcome through molecular profiling in stage III melanoma. Clin. Cancer Res. 14: 5173-80 (2008).

17 Jonsson G, Busch C, Knappskog S et al. Gene expression profiling-based identification of molecular subtypes in stage IV melanomas with different clinical outcome. Clin. Cancer Res. 16, 3356-3367 (2010).

18 Mann GJ, Pupo GM, Campain AE et al. $B R A F$ mutation, NRAS mutation, and the absence of an immune-related expressed gene profile predict poor outcome in patients with stage III melanoma. J. Invest. Dermatol. 133, 509-517 (2013).

19 Parker JS, Mullins M, Cheang MC et al. Supervised risk predictor of breast cancer based on intrinsic subtypes. J. Clin. Oncol. 27, 1160-1167 (2009).

20 Ulloa-Montoya F, Louahed J, Dizier B et al. Predictive gene signature in MAGE-A3 antigen-specific cancer immunotherapy. J. Clin. Oncol. 31, 2388-2395 (2013).

21 Ji RR, Chasalow SD, Wang L et al. An immune-active tumor microenvironment favors clinical response to ipilimumab. Cancer Immunol. Immunother. 61, 1019-1031 (2012).

22 Harbst K, Staaf J, Lauss M et al. Molecular profiling reveals low- and high-grade forms of primary melanoma. Clin. Cancer Res. 18, 4026-4036 (2012).

23 Harbst K, Lauss M, Cirenajwis H et al. Molecular and genetic diversity in the metastatic process of melanoma. J. Pathol. 233, 39-50 (2014).

24 Van Allen EM, Wagle N, Sucker A et al. The genetic landscape of clinical resistance to raf inhibition in metastatic melanoma. Cancer Discov. 4, 94-109 (2014). 